\title{
Alternating current conduction properties of thermally evaporated $\alpha$-nickel phthalocyanine thin films: Effects of oxygen doping and thermal annealing
}

\author{
Thomas D. Anthopoulos ${ }^{\mathrm{a})}$ and Torfeh S. Shafai \\ Staffordshire University, School of Engineering and Advanced Technology, Beaconside, \\ Stafford ST18 ODF, United Kingdom
}

(Received 18 February 2003; accepted 28 May 2003)

\begin{abstract}
The ac conduction properties of thermally evaporated films of $\alpha$-nickel phthalocyanine $(\alpha$-NiPc) were studied in situ and ex situ employing symmetric gold ohmic electrodes in the frequency range of $20-10^{6} \mathrm{~Hz}$ at various temperature regimes. ac conductivity was identified to be via a hopping-type mechanism in the lower temperature region and via a free-band conduction in the high temperature region. Upon exposure of the films to dry air, the low frequency ac conductivity was found to increase by 2 orders of magnitude, which was attributed to oxygen absorption within NiPc. The doping effect was partially reversed by thermal annealing of the films under high vacuum. Measurements on the dependence of capacitance and loss tangent $(\tan \delta)$ on frequency were consistent and quantitatively explained by invoking an equivalent circuit model. Oxygen doping was found to increase the low frequency capacitance of NiPc. The phenomenon was understood in terms of reduction in the value of device internal resistance induced by oxygen absorption. (C) 2003 American Institute of Physics. [DOI: 10.1063/1.1592626]
\end{abstract}

\section{INTRODUCTION}

Understanding the electronic charge transport processes in small molecule organic materials such as phthalocyanines (Pcs) is interesting both from fundamental and technological point of view. In recent years, owing to their very interesting physical properties, this particular family of materials has raised considerable deal of research interest followed by technological applications in the field of micro/ optoelectronics. ${ }^{1-3}$ Significant research efforts have been focused to the study of the charge transport properties of these compounds in thin film formation and the relation of film microstructure on the macroscopic properties of devices made out of them. ${ }^{4}$ Such understanding is essential for improving the quality and performance of electronic devices like organic light-emitting diodes, photovoltaic cells, and organic field-effect transistors. Among many electrical impedance analysis has proved to be a powerful technique for identifying and characterising the charge transport phenomenon in various materials. Though there are numerous reports on the direct current $(\mathrm{dc})$ electrical properties of phthalocyanines based thin films, ${ }^{4-6}$ very little work has been carried on the alternating current (ac) electrical transport phenomena of these materials and the effect of doping with oxygen. This article presents a study on the ac conduction properties and dielectric loss factor tangent of thermally evaporated $\alpha$-nickel phthalocyanine ( $\alpha$-NiPc) films. Particular emphasis is placed on the effects of oxygen doping and thermal annealing of the as-evaporated films.

\footnotetext{
${ }^{a)}$ Present and corresponding address: Department of Physics and Astronomy, University of St. Andrews, North Haugh, St. Andrews, Fife KY16 9SS, United Kingdom; electronic mail: ta10@st-andrews.ac.uk
}

\section{EXPERIMENTAL DETAILS}

Nickel phthalocyanine (Sigma-Aldrich Ltd) was further purified by entrainer sublimation technique. The entire process was carried under atmospheric pressure using highly pure nitrogen as the carrier gas. Purified NiPc was obtained in the cooler zone as long needle-like crystals $\leqslant 3 \mathrm{~cm}$ long, $\leqslant 0.1 \mathrm{~cm}$ wide, and $\leqslant 0.02 \mathrm{~cm}$ thick. NiPc was chosen because it is known to exhibit a relatively high value of hole mobility. ${ }^{7,8}$ The molecular structure of NiPc is shown in the inset of Fig. 1. Structural properties of NiPc were studied in the form of (i) purified single crystals; (ii) as-evaporated thin films; and (iii) annealed films, by means of x-ray diffractometry (XRD). The XRD patterns were recorded in a Phillips 1050 diffractometer using $\mathrm{Cu} K \alpha$ irradiation. The $2 \theta$ scan range was varied from 5.0 to 35.0 with a generator current of $35 \mathrm{~mA}$ at $40 \mathrm{kV}$, an angular increment of $0.02^{\circ}$ and a time interval of $4 \mathrm{~s} / \mathrm{step}$. Accurate determination of the peak intensities was obtained by employing line-profiling analysis. For electrical characterization, sandwich structure devices consisting of gold/ $\alpha$-NiPc/gold were prepared in situ by thermal sublimation under high vacuum $\left(10^{-4} \mathrm{~Pa}\right)$. Gold was chosen because it is known to provide good and reproducible ohmic contacts with many phthalocyanines including NiPc. ${ }^{4,5}$ Details of the fabrication method employed have been reported earlier. ${ }^{9}$ The active conduction area of each device $(A)$ was measured after fabrication to be approximately $1.2 \times 10^{-5} \mathrm{~m}^{2}$. Electrical characterization was performed in situ and ex situ employing a HP 4884A precision LCR meter under light tight conditions. The effects of temperature on the conduction mechanism were investigated using a cryostat system designed to maintain constant low pressure for temperatures in the range $180-550 \mathrm{~K}$. In order to study the influence of oxygen as a dopant molecule, the 


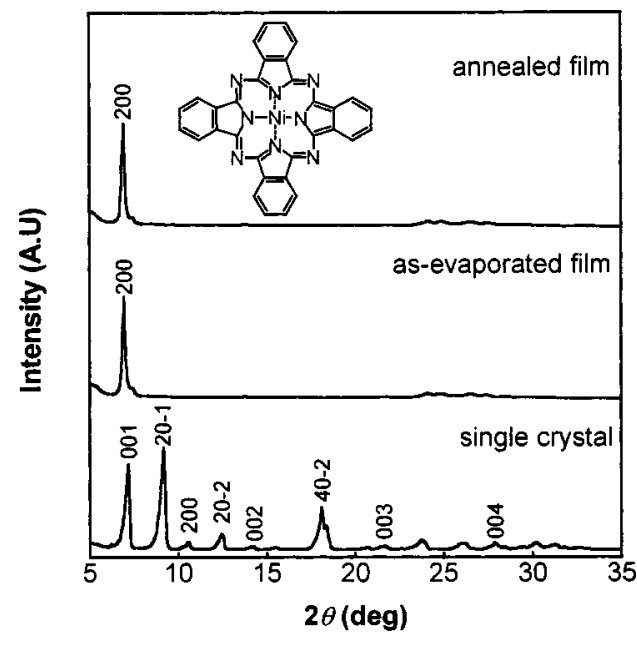

FIG. 1. Typical x-ray diffraction patterns of NiPc in the form of single crystals, as-deposited and annealed film. Indexing of the peaks for the single crystal was performed assuming a monoclinic type structure, whereas in the case of thin film considering a tetragonal type system.

freshly prepared devices were transferred into a desiccator system and exposed to dry air for prolonged period of time.

\section{RESULTS AND DISCUSSION}

\section{A. Structural characterization of NiPc}

Typical x-ray diffraction patterns of NiPc obtained in the form of single crystals, as-deposited, and annealed films are shown in Fig. 1. For the single crystal trace two distinct peaks are visible at low diffraction angles. The latter exhibits similar characteristics with the traces reported previously for $\beta$-nickel phthalocyanine ( $\beta$-NiPc) powder ${ }^{10}$ and $\beta$-copper phthalocyanine $\left(\beta\right.$-CuPc) single crystals. ${ }^{11}$ Hence, the single crystals obtained in the present study can also be identified to be of the stable $\beta$-polymorph. Indexing of the films was successfully performed assuming a monoclinic crystalline system $^{12}$ in which the three crystal axes are of unequal lengths, having one of their intersections oblique and the other two at right angles. ${ }^{13}$ In the contrary, for the asevaporated and annealed films a single strong reflection peak is observed with experimental data being in better agreement with the tetragonal rather than the monoclinic crystal structure. From the full-width and half maximum of the first diffraction peak, for both as-deposited and annealed film, an estimate of the crystalline cluster approximately $32.5 \mathrm{~nm}$ was calculated using the Scherrer equation. This value falls within the range of previously reported values of $5.3 \mathrm{~nm}$ for $\mathrm{PbPc},{ }^{14} 28.8 \mathrm{~nm}$ for $\mathrm{CuPc},{ }^{11}$ and $100 \mathrm{~nm}$ for NiPc. ${ }^{15}$ Discrepancy between the latter and the value derived in the present work is probably attributed to different deposition conditions. From close examination of Fig. 1, it is evident that the as-evaporated $\alpha$-NiPc film remain virtually unaltered upon thermal annealing $(517 \mathrm{~K})$, implying that no phase transition has taken place nor the characteristic preferential crystalline orientation (200) has changed.

\section{B. ac conductivity measurements}

ac spectroscopic measurements have been obtained in situ for several $\mathrm{Au} / \alpha$-NiPc/Au devices in the frequency range

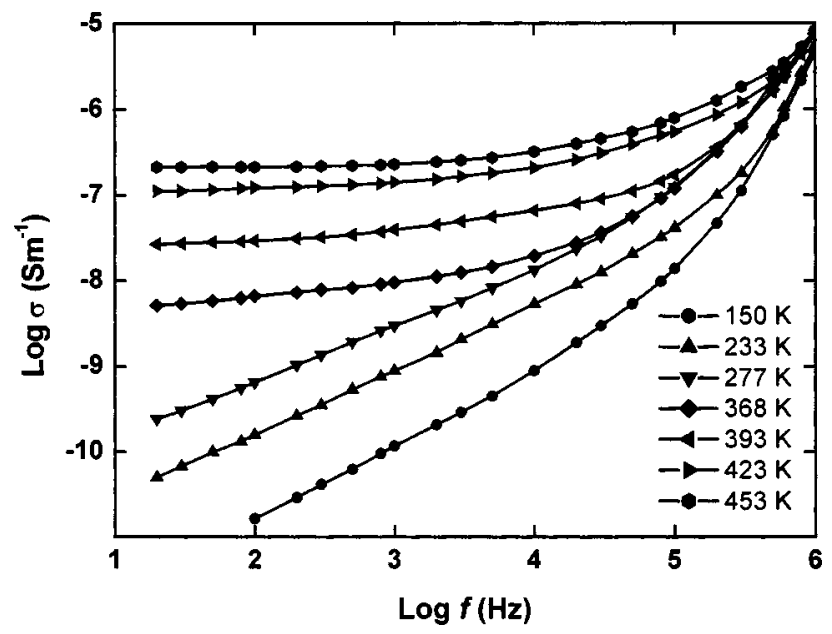

FIG. 2. Dependence of conductivity $(\sigma)$ as a function of frequency $(f)$ for an $\mathrm{Au} / \alpha-\mathrm{NiPc} / \mathrm{Au}$ device measured at different temperatures.

of $20-10^{6} \mathrm{~Hz}$ utilizing an oscillating voltage with amplitude $100 \mathrm{mV}$ peak-to-peak. Figure 2 shows several logarithmic representations of the ac conductivity dependence $(\sigma)$ on frequency $(f)$ at different temperatures in the range 150-423 K. Thickness of the $\alpha$-NiPc layer employed for this experiment was $0.5 \mu \mathrm{m}$. It can be seen from this figure that for temperatures below $300 \mathrm{~K}$, conductivity becomes frequency dependent. Within this temperature region and for a wide range of frequencies $\left(\sigma_{\mathrm{AC}}\right)$ takes the form of a power law ${ }^{16}$

$$
\sigma_{\mathrm{AC}}(\omega, T)=A \omega^{S}
$$

where $\sigma_{\mathrm{AC}}$ is the ac conductivity, $A$ is a constant, $\omega$ is the angular frequency $(=2 \pi f)$, and $s$ is the exponent of the frequency component. For temperatures $T>300 \mathrm{~K}$, conductivity is nearly constant for a wide frequency range (20 $-10^{4} \mathrm{~Hz}$ ) up to a threshold frequency at which starts rising steeply. In the low frequency range $\sigma_{\mathrm{AC}}$ is also strongly dependent on the absolute temperature while for high frequencies $(1 \mathrm{MHz})$ it becomes temperature independent. This behavior is possibly attributed to the time constant of the high frequency ac stimulation which is too short for charge carriers to relax, thus, giving rise to the temperature independent conductivity. Results qualitatively similar to those presented in Fig. 2 have been reported previously for pellets of NiPc maintained under vacuum. ${ }^{17}$ In the latter study the band model was proposed for high temperature low-frequency region, while hopping-type conduction for the low-temperature high-frequency region. Generally, decrease of conductivity with increasing frequency is associated with a band-type conduction process, while increase with a hopping-type conduction mechanism. ${ }^{18,19}$ In addition to the gradual increase of conductivity as a function of frequency the value of index $s$ has also been found to decrease with increasing temperature. In particular, for low frequency and low temperature regime the exponent $s$ increases linearly with decreasing temperature as shown in Fig. 3. This behavior is consistent with the correlated barrier hopping $(\mathrm{CBH})$ model for carriers over the potential barrier separating two centres in a random distribution. ${ }^{16}$ In the latter model the temperature dependence of the frequency exponent $s$ can be expressed as ${ }^{16}$ 


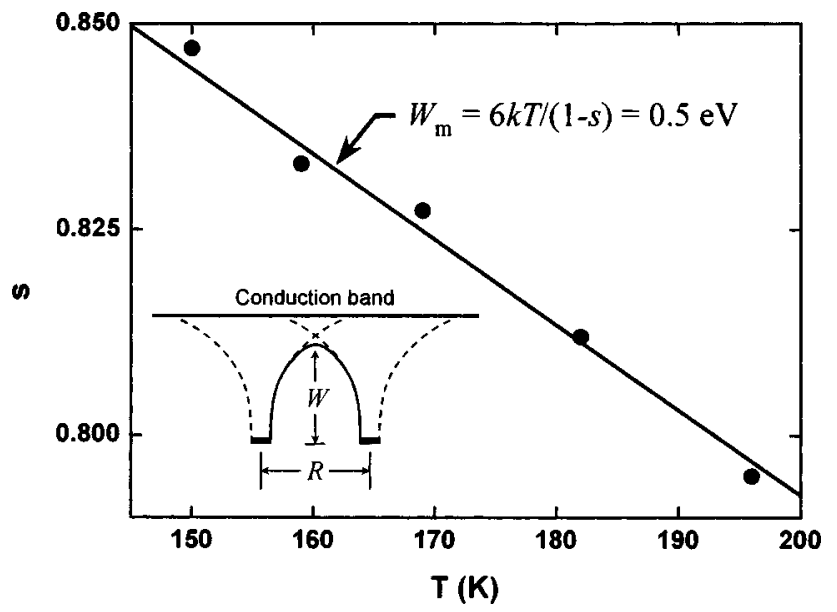

FIG. 3. Temperature dependence of index $(s)$ measured for an $\mathrm{Au} / \alpha$ $\mathrm{NiPc} / \mathrm{Au}$ device in the frequency range $100-10^{4} \mathrm{~Hz}$. Inset illustrates the lowering of the barrier height for two closely spaced charged centres in the $\mathrm{CBH}$ model employed.

$$
s=1-\frac{6 k T}{W_{m}},
$$

where $W_{m}$ is the effective potential barrier at infinite intersite separation $(R=\infty)$, which, for the case of two-electron transition, is given as

$$
W_{m}=\frac{2 e^{2}}{\pi \varepsilon \varepsilon_{0} R}+W .
$$

In Eq. (3), $\varepsilon \varepsilon_{0}$ is the permittivity of the semiconductor $\left(\sim 2.42 \times 10^{-11} \mathrm{~F} \mathrm{~m}^{-1}\right.$ for $\left.\mathrm{NiPc}\right),{ }^{5} R$ is the separation between the neighboring sites, and $W$ the hopping barrier potential after lowering of the effective barrier $\left(W_{m}\right)$ due to Coulomb wells overlap. By substituting Eq. (3) into Eq. (2) the following expression for the hopping distance $(R)$ can be obtained:

$$
R=\frac{2 e^{2}}{\pi \varepsilon \varepsilon_{0}\left(\frac{6 k T}{1-s}-W\right)} .
$$

As shown in Fig. 3, the linear increase of $s$ is in good agreement with the $\mathrm{CBH}$ model described by Eq. (2) employing a value for $W_{m}=0.5 \mathrm{eV}$. Substituting the lowest and highest activation energy for the dc conductivity values of 10 and $300 \mathrm{meV}$ (data not shown), respectively, for the hopping barrier potential $(W)$ in Eq. (4) (see inset of Fig. 3), the hopping distance is found to lie within the range 8.5-20 nm. Both values appear to be smaller than the size of the crystalline cluster $(\sim 32.5 \mathrm{~nm})$ implying a charge carrier hopping process between localized sites within the cluster.

The maximum value for $s$ obtained in the present work is $\sim 3.3$ for the frequency range $0.5-1 \mathrm{MHz}$ at $150 \mathrm{~K}$. The latter is higher than index values reported previously $(<1)$ for various other metal phthalocyanines. ${ }^{19-21}$ However, Ray et al $^{14}$ have reported a nearly quadratic dependence of conductivity on frequency $(1 \mathrm{MHz})$ for partially monoclinic films of $\mathrm{PbPc}$. In the latter study, experimental results were attributed to the metal/semiconductor contact rather than the

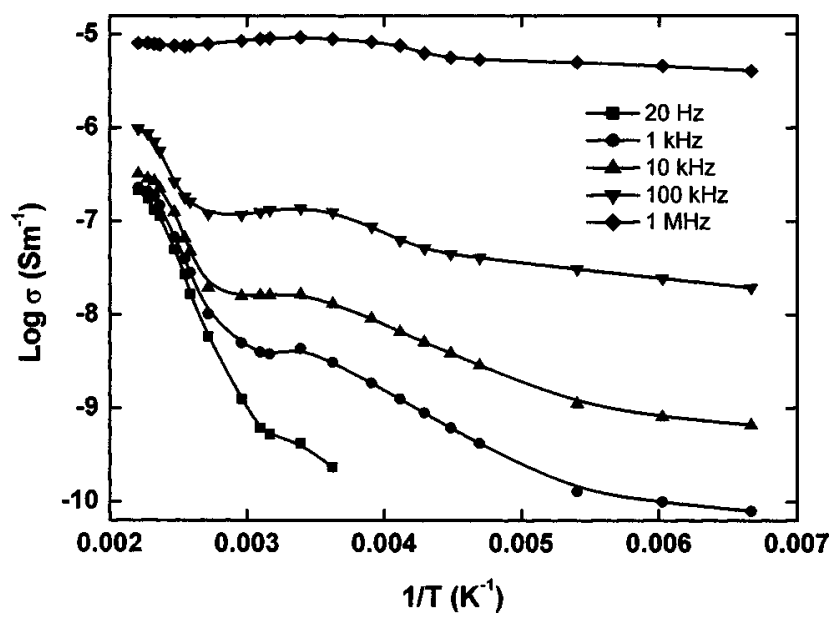

FIG. 4. Temperature dependence of ac conductivity $(\sigma)$ in a thin film of $\alpha$-NiPc maintained under high vacuum.

bulk properties of PbPc. In the present study this extraordinary high value of $s$ is believed to be due to resonance effects of the test circuit rather than the bulk properties of NiPc. Contact effects between $\mathrm{Au}$ and $\alpha$-NiPc are also excluded since electrical conduction through similar devices has been shown to be a bulk rather than an electrode limited process. ${ }^{5}$

\section{1. ac conductivity as a function of temperature}

The variation of ac conductivity as a function of temperature in thin films of $\alpha-\mathrm{NiPc}$ has also been studied for the same device samples. Figure 4 shows the semilogarithmic plot of $\sigma$ versus $1 / T$ at five different frequencies in the temperature range $150-423 \mathrm{~K}$. Three different conduction regions are evident for three different temperature ranges: (i) $T<185 \mathrm{~K}$; (ii) $210<T<185 \mathrm{~K}$; and (iii) $360<T<420 \mathrm{~K}$. Activation energies were calculated for all three temperature regimes. Derived values are tabulated in Table I. At low temperatures $(T<180 \mathrm{~K})$ conductivity is largely dependent upon frequency. This dependence is found to decrease with increasing temperature. At low temperatures conductivity becomes practically temperature independent exhibiting very low activation energies. The value for activation energy of charge carriers calculated at $T<180 \mathrm{~K}$ falls within the range of 14-25 meV, thereby providing further evidences for the existence of a hopping type conduction mechanism. Although, this value is somewhat higher than the activation energy value of $12 \mathrm{meV}$ for thin films of $\mathrm{PbPc},{ }^{14}$ it falls

TABLE I. Activation energy values for the ac conductivity derived at three different temperature ranges at different frequencies for an $\mathrm{Au} / \alpha-\mathrm{NiPc} / \mathrm{Au}$ sample.

\begin{tabular}{lccc}
\hline \hline & \multicolumn{3}{c}{ Activation energy (eV) } \\
\cline { 2 - 4 } Frequency $(\mathrm{Hz})$ & $T<180 \mathrm{~K}$ & $210<T<180 \mathrm{~K}$ & $360<T<420 \mathrm{~K}$ \\
\hline 20 & - & - & 0.65 \\
$10^{3}$ & 0.02 & 0.16 & 0.6 \\
$10^{4}$ & 0.025 & 0.124 & 0.55 \\
$10^{5}$ & 0.025 & 0.1 & 0.36 \\
$10^{6}$ & 0.014 & 0.05 & - \\
\hline \hline
\end{tabular}




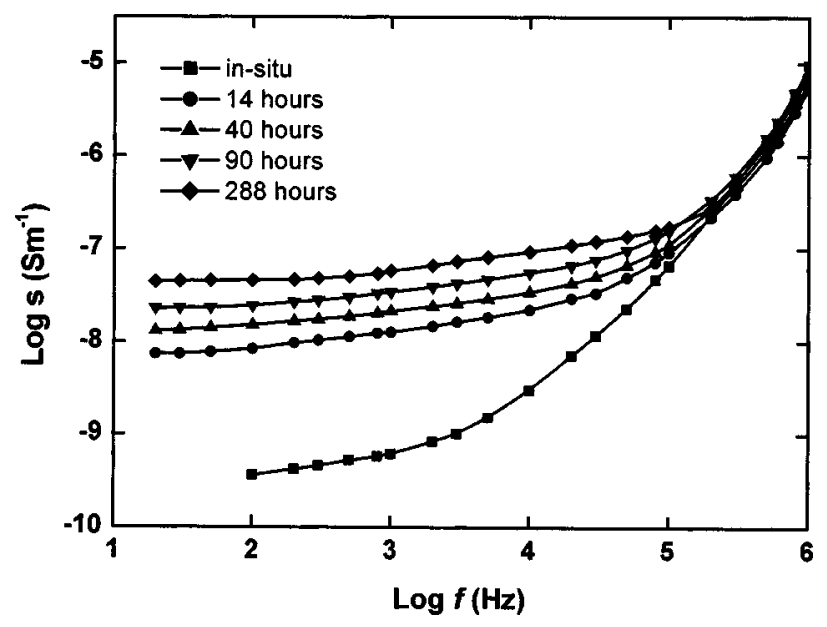

FIG. 5. Dependence of ac conductivity $(\sigma)$ on frequency $(f)$ at different exposure times to dry air for thin films of $\alpha$-NiPc.

within the range of previously reported values of 15 and 42 $\mathrm{meV}$, for freshly prepared and heat treated films of cobalt phthalocyanine $(\mathrm{CoPc})$, respectively. ${ }^{20}$ At temperatures above $210 \mathrm{~K}$ a transition on the conduction mechanism occurs. Activation energy values obtained from this region vary from 160 to $50 \mathrm{meV}$. It is evident from Table I that activation energy is strongly frequency dependent. Conductivity at this temperature region is usually attributed to impurity centres located at different energy levels within the forbidden energy band gap of the material. ${ }^{18}$ The break in the linear-type dependence of $\sigma$ versus $1 / T$, observed for $289<T<360 \mathrm{~K}$, is most probably a result of oxygen molecule desorption as a result of increasing temperature. ${ }^{22}$ The characteristic temperature at which desorption peaks occur $(280<T<360 \mathrm{~K})$ it seems to be in a good agreement with the desorption peak temperature reported previously for as-evaporated films of NiPc. ${ }^{7}$ Finally, at even higher temperatures $(T>360 \mathrm{~K})$ another linear region in the $\sigma$ versus $1 / T$ curve is observed. The only exemption is the characteristic obtained at $1 \mathrm{MHz}$, where no activation energy has been identified. The linearity of this region is attributed to intrinsic rather extrinsic conductivity since at these temperatures any absorbed $\mathrm{O}_{2}$ molecules, are expected to be completely outgassed. These relatively high value activation energies suggest that free-band type is the most likely charge transfer mechanism that dominates conduction at this temperature range.

\section{Effects of oxygen doping on ac conductivity}

It well established that the effect of oxygen doping on dc conductivity of Pc based devices is drastic., ${ }^{4,9,23}$ In order to identify whether the effects of $\mathrm{O}_{2}$ doping are similarly pronounced in the case of ac conductivity, a sample consisting of a $0.4 \mu \mathrm{m}$ thick NiPc layer has been realized. Figure 5 shows a logarithmic plot of $\sigma$ versus $f$ for various exposure times to dry air. It is evident that in the low frequency range conductivity is strongly dependent on exposure time. However, for frequencies $f>10^{5} \mathrm{~Hz}$ this dependence is diminished. A better graphical representation of the dependence between these two variables is shown in Fig. 6. As can be seen at a frequency of $1 \mathrm{MHz}$, conductivity shows no change

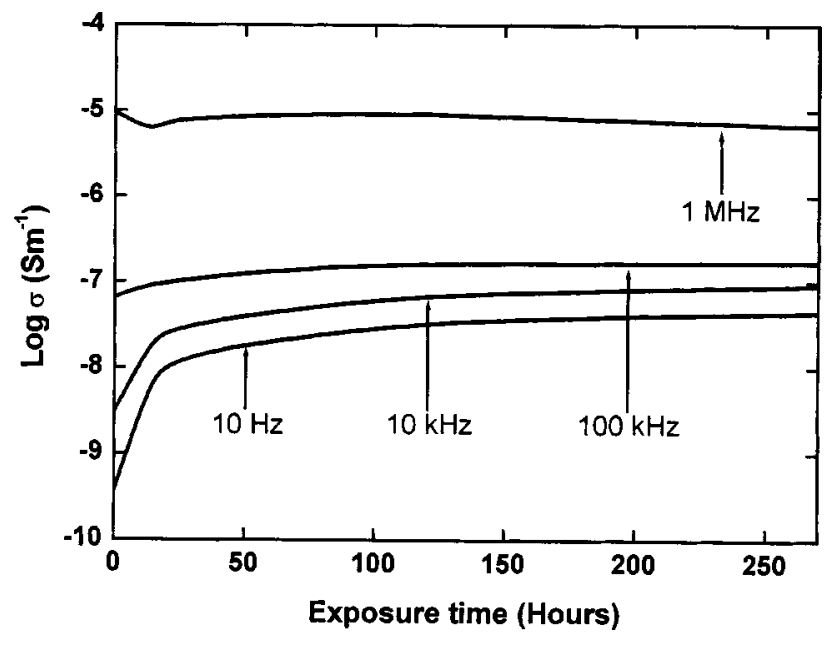

FIG. 6. Dependence of ac conductivity $(\sigma)$ in thin films of $\alpha$-NiPc on exposure time to dry air at different frequency levels.

as a function of exposure time. On the contrary, at low frequencies, such as $100 \mathrm{~Hz}$ (at which $\sigma_{\mathrm{AC}} \approx \sigma_{\mathrm{DC}}$ ), conductivity increases markedly. Since dc conductivity within $p$-type semiconductor materials can be expressed as

$$
\sigma_{\mathrm{DC}}=p_{0} e \mu,
$$

an observed increase in $\sigma$ is attributed to the increase of hole concentration $\left(p_{0}\right)$ due to oxygen doping. Furthermore, at a frequency of $1 \mathrm{MHz}$ conductivity is approximately $10^{-5} \mathrm{~S} \mathrm{~m}^{-1}$. This large value is possibly due to elimination of certain resistance at the contacts between microcrystalline regions. Such resistive elements, if large, will be electrically shorted by the capacitance of the contact. ${ }^{24}$

\section{Effects of heat-treatment on ac conductivity}

Measurements of $\sigma$ vs $f$ were obtained: (i) under in situ conditions; (ii) after exposure to dry air for $288 \mathrm{~h}$; and (iii) after heat treatment of the sample in high vacuum at $395 \mathrm{~K}$ for $10 \mathrm{~min}$. These results are presented in Fig. 7. As can be seen, a decrease in conductivity by almost a factor of 27 (when compared with conductivity value obtained for the

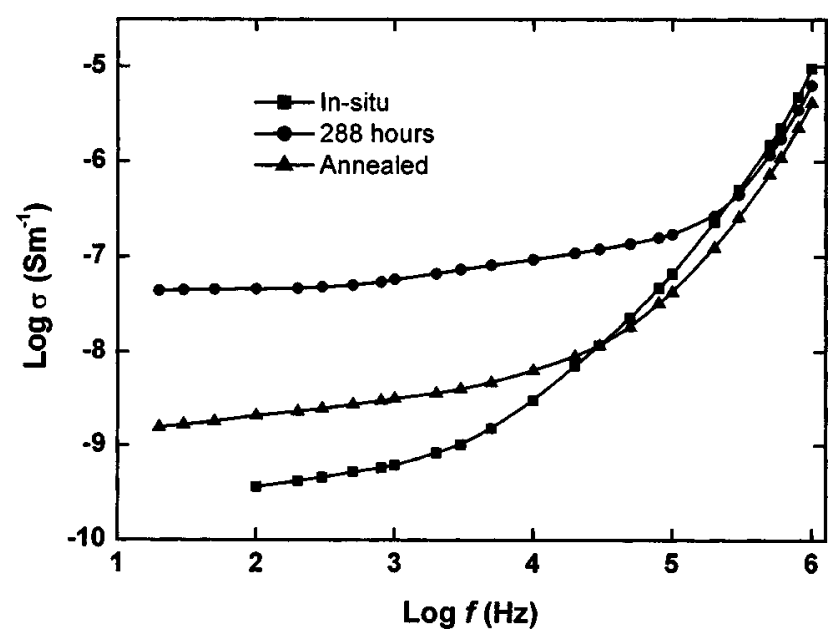

FIG. 7. Dependence of ac conductivity $(\sigma)$ on frequency $(f)$. Under in situ, after exposure to dry air, and after heat treatment $(395 \mathrm{~K})$ in high vacuum. 


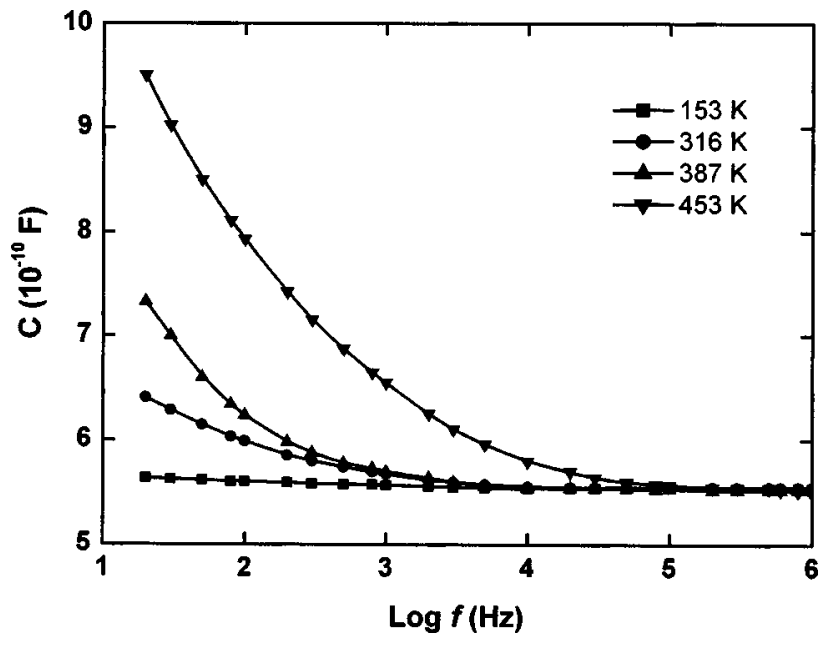

FIG. 8. Capacitance $(C)$ dependence on frequency $(f)$ for $\alpha$-NiPc films obtained at different temperatures.

sample exposed to dry air for $288 \mathrm{~h}$ ) is observed. Gould and Hassan ${ }^{19}$ have also observed similar reduction in conductivity for heat-treated films of $\mathrm{CuPc}$. This effect is strongly believed to be associated with desorption of $\mathrm{O}_{2}$ molecules from the NiPc layer. Nevertheless, complete removal of oxygen was not achieved since the measured conductivity is substantially higher when compared with the value obtained for the in situ device. Such behavior can be explained in terms of: (i) the relatively low annealing temperature (395 K); and (ii) the short duration of the annealing process $(10 \mathrm{~min})$. In the case of chlorine doped PbPc films, Abass et al. ${ }^{25}$ have shown that chlorine release is a function of both annealing temperature and time.

\section{Capacitance as a function of frequency and temperature}

The effects of frequency and temperature on the capacitance of $\mathrm{Au} / \alpha$-NiPc/Au devices were also investigated. Figure 8 displays the variation of capacitance with frequency at various temperatures for a NiPc film of thickness $0.5 \mu \mathrm{m}$. As can be seen, at low temperatures the capacitance is nearly constant throughout the entire frequency spectrum investigated. However, as temperature increases the low frequency range capacitance increases markedly. For frequencies $>50 \mathrm{kHz}$ capacitance becomes temperature independent and approaches a common value of $560 \mathrm{pF}$. Similar behavior has been observed for $\mathrm{CuPc}^{19}$ and $\mathrm{PbPc}$ films. ${ }^{21}$ The drastic increase of capacitance in the high temperature-low frequency range is probably due to space charge polarization induced by the increasing number of free carriers as a result of increasing temperature. ${ }^{26,19}$ This behavior is better demonstrated in Fig. 9 where the capacitance of NiPc based devices is plotted as a function of temperature for different frequency levels. For $T<220 \mathrm{~K}$, capacitance varies slightly with temperature for any signal frequency. As temperature rises above $220 \mathrm{~K}$ capacitance becomes frequency dependent for $f$ $>1 \mathrm{kHz}$. At temperatures between 290 and $350 \mathrm{~K}$, capacitance exhibits a peak value followed by a decrease around $300-365 \mathrm{~K}$, above which another increase is observed. The

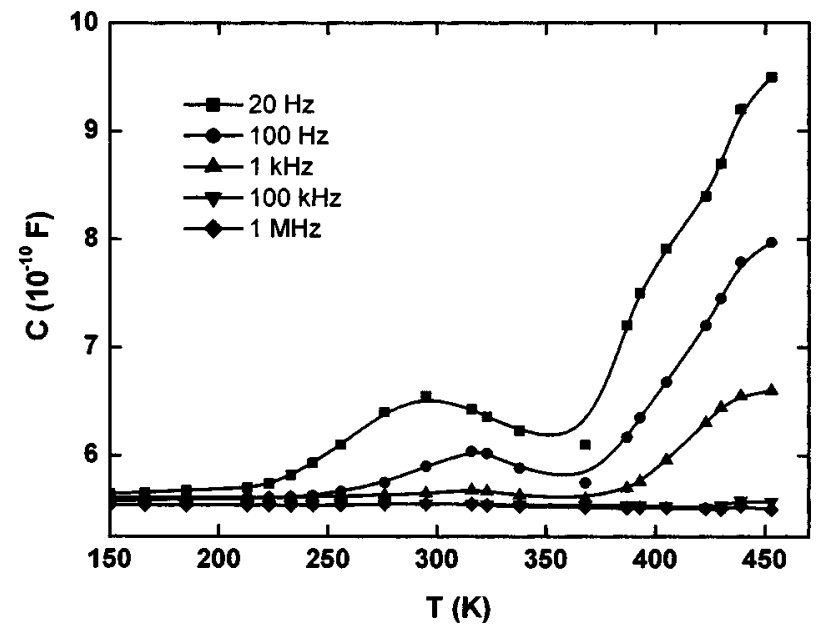

FIG. 9. Variation of capacitance $(C)$ in $\alpha$-NiPc films as a function of temperature for different frequency levels.

appearance of the first maxima can be ascribed to absorbed oxygen molecules within NiPc that tend to desorb at higher temperatures. Similar observations have been reported for thin films of $\mathrm{CoPc}^{20}$ The observed increase of capacitance with temperature, and the subsequent decrease with frequency, may be qualitatively explained by the equivalent circuit model developed by Goswami and Goswami ${ }^{27}$ for $\mathrm{ZnS}$ films employing ohmic contacts. According to this model the measured capacitance $\left(C_{S}\right)$ is given by

$$
C_{S}=C+\frac{1}{\omega^{2} R^{2} C},
$$

where $\omega$ is the angular frequency, $C$ is a frequency independent capacitance element, and $R$ the interior resistance of the material given as

$$
R=R_{0} \exp \left(\frac{\Delta E}{k T}\right)
$$

where $R_{0}$ is a constant and $\Delta E$ the activation energy. In Eq. (7) an increase in the value of $T$ implies a decrease of $R$ and hence an increase in the value of $C_{S}$ from Eq. (6). This prediction is clearly evident in Figs. 8 and 9. Furthermore, Eq. (6) also predicts that the measured capacitance should decrease with increasing frequency reaching a value of $C$ for $f=\infty \mathrm{Hz}$. This effect is confirmed in the $C$ versus $f$ plot shown in Fig. 8, providing further experimental evidence on the validity of this model.

\section{Loss tangent as a function of frequency and temperature}

For an ideal parallel plate capacitor no energy losses should occur and the current should lead the applied voltage by exactly $90^{\circ}$. In reality the total current transversing such a capacitor is inclined by a power factor angle $\theta<90^{\circ}$ against the applied voltage $V$. The main reason causing this type of behavior is the existence of internal capacitor resistance $(R)$ (for an ideal capacitive element $R=0 \Omega$ ) that leads to dissipation of power. Under these circumstances the phase angle between current and voltage will be less than $90^{\circ}$ and the loss factor $(\tan \delta)$ can be defined by 


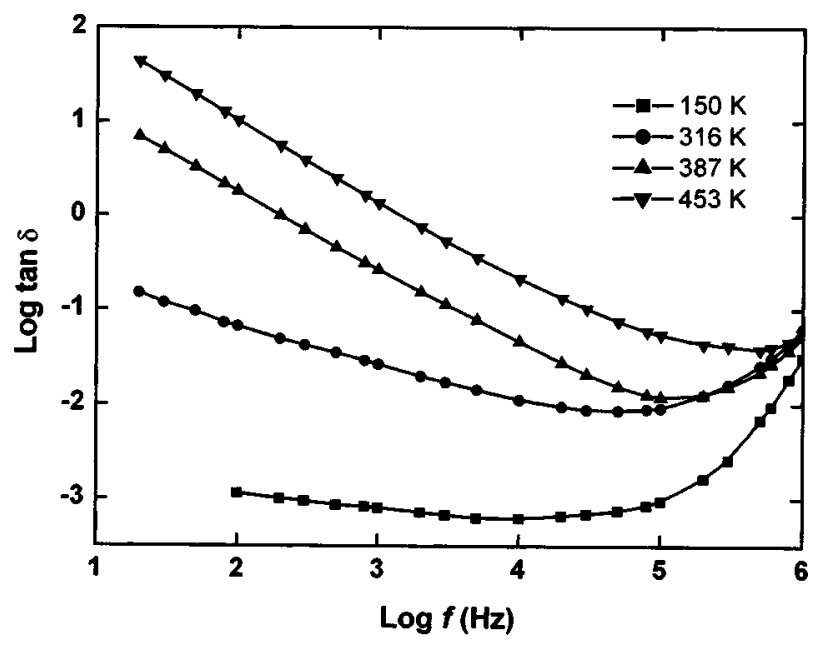

FIG. 10. Variation of loss factor $(\tan \delta)$ as a function of frequency $(f)$ evaluated at different temperatures.

$$
\tan \delta=\frac{1}{\omega R C_{S}},
$$

or

$$
\tan \delta=\frac{G}{\omega C_{S}},
$$

where $C_{S}$ is the measured capacitance and $G$ the conductance of the device. Figure 10 illustrates the variation of $\tan \delta$ as a function of frequency for a NiPc device at various temperatures. It is apparent from this figure that $\tan \delta$ decreases with increasing frequency while $\delta$ increases with increasing temperature. The common feature, which characterizes each curve in this figure, is the presence of a minimum. The latter observation agrees with the previously reported characteristics for $\mathrm{ZnS}^{27}$ and $\mathrm{CoPc}^{20}$ films. The model of Goswami and Goswami $^{27}$ may also explain such behavior. According to this model, the loss tangent $(\tan \delta)$ is given by

$$
\tan \delta=\frac{\left(1+\frac{r}{R}\right)}{\omega R C}+\omega r C,
$$

where $r$ is a series resistance due to leads (usually frequency and temperature independent) and $C$ the frequency independent inherent capacitive element. From Eq. (10) it is evident that at low frequencies $\tan \delta$ will decrease with increasing frequency ( $\omega^{-1}$ dominant term) followed by a loss minimum and subsequent increase in the high frequency range $(\omega$ dominant term). The angular frequency at which the loss minimum will be observed is given by

$$
\omega_{\min }=\frac{1}{C \sqrt{r R}} .
$$

Since $R$ is expected to decrease with increasing temperature the value of $\omega_{\min }$, according to Eq. (11), will rise. This effect is demonstrated in Fig. 11 where the $\omega_{\text {min }}$ is plotted versus temperature, thus, providing further experimental evidence on the validity of this model. For large values of $\omega$ (i.e., $f$ $=1 \mathrm{MHz}$ ), Eq. (10) reduces to

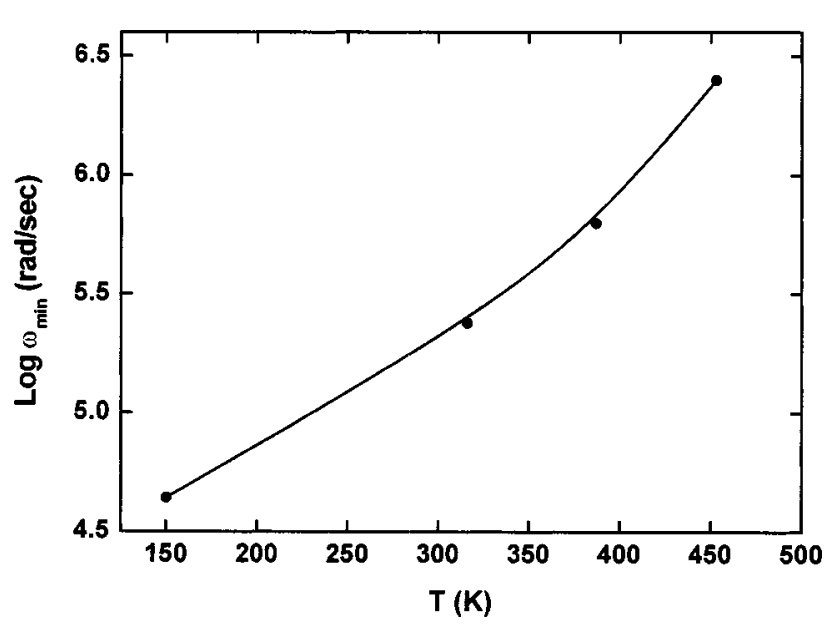

FIG. 11. Dependence of $\omega_{\min }$ on absolute temperature $(T)$ for a $\alpha$-NiPc film.

$$
\tan \delta=\omega r C
$$

since $R \gg r$ in all cases. Now utilizing the high frequency capacitance value $(560 \mathrm{pF})$ together with Eq. (12), analysis of the data in Fig. 10 yields a value for $r=5.68 \Omega$. This value is in good agreement with the independently measured value of $r=5.1 \Omega$ using a HP ohmmeter. Another prediction of Eq. (10) is the increase of $\tan \delta$ with increasing temperature since $R$ reduces. This prediction is confirmed in the $\tan \delta$ versus $T$ dependence shown in Fig. 12. As can be seen anomalous behavior is observed for all frequency levels in the temperature range of $280-360 \mathrm{~K}$ and is possibly attributed to outgassing of oxygen from the NiPc layer.

\section{Effects of oxygen doping and annealing on capacitance}

The variation of capacitance $(C)$ with frequency $(f)$ has been studied for several NiPc films of thickness $0.4 \mu \mathrm{m}$ as a function of exposure time to dry air. Obtained results are presented in Fig. 13. The plots show that for low frequencies capacitance changes markedly with exposure time. However, for frequency values $f>30 \mathrm{kHz}$, capacitance becomes nearly frequency independent and reaches a minimum value of

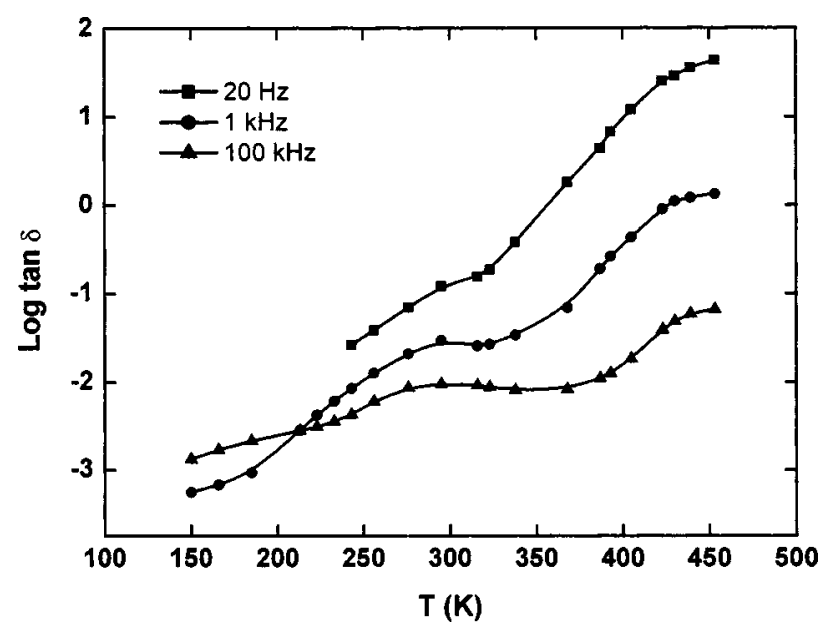

FIG. 12. Temperature dependence of $\operatorname{loss}$ factor $(\tan \delta)$ obtained at different frequencies. 


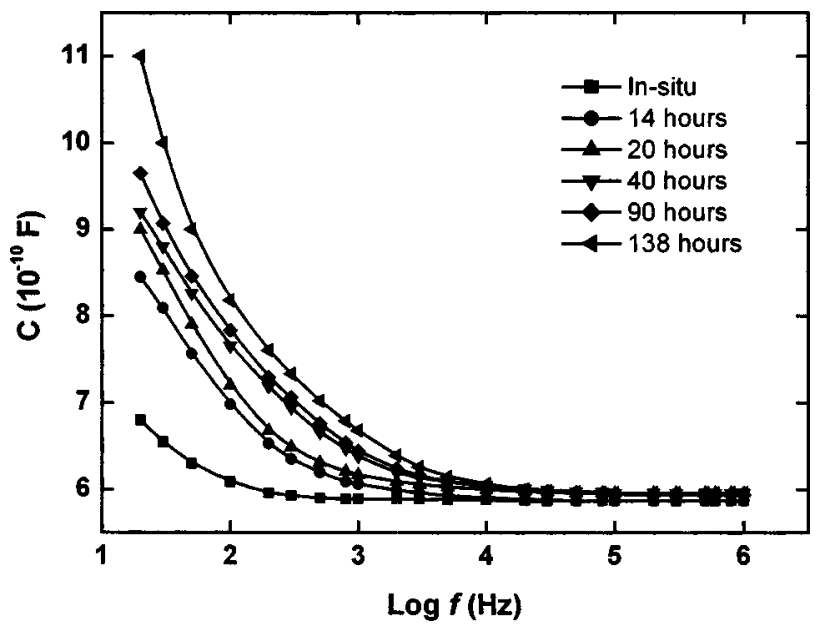

FIG. 13. Dependence of capacitance $(C)$ on frequency $(f)$ at different exposure times of the $\alpha$-NiPc film to dry air.

$\sim 600 \mathrm{pF}$. The increase of $C_{S}$ in the low frequency range of Fig. 13 is attributed to increase in the conductivity of $\mathrm{NiPc}$ layer induced by oxygen doping leading to a decrease in the value of $R$ in Eq. (6), and consequently increase the measured capacitance. Similar observations have been reported in the literature for thin films of PbPc. ${ }^{21}$ An additional factor that may also lead to increase in the value of the measured capacitance is the increase of polarization. Such increase can result from absorption of polar molecules such as water molecules. In the latter case the oxygen atom has a greater electronegativity than the hydrogen atoms, therefore, under the influence of external electric field water molecules are expected to contribute to the measured capacitance by their permanent dipole moment.

The effects of heat treatment on $C$ versus $f$ characteristics of NiPc were also studied. Annealing process was performed under high vacuum at $395 \mathrm{~K}$ and lasted approximately $10 \mathrm{~min}$. Sample devices were then cooled down to room temperature under vacuum prior to electrical characterization. Figure 14 presents the $C$ versus $f$ plots for an $\mathrm{Au} / \alpha$ -

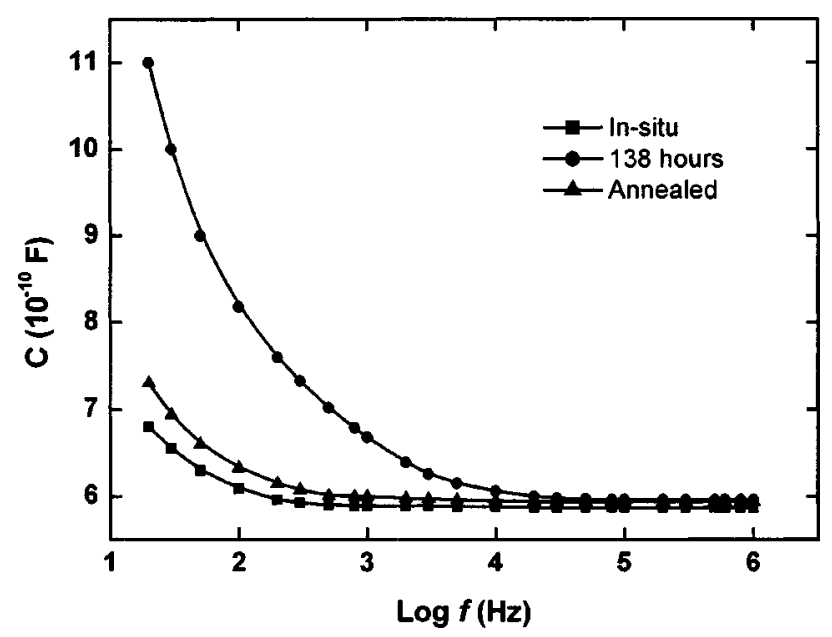

FIG. 14. Dependence of capacitance $(C)$ on frequency $(f)$ for an $\mathrm{Au} / \alpha$ $\mathrm{NiPc} / \mathrm{Au}$ device obtained under in situ, after exposure to dry air for $138 \mathrm{~h}$, and after heat treatment of the film $(395 \mathrm{~K})$ in high vacuum.
NiPc/Au sample obtained under: (i) in situ, (ii) after exposure to dry air for $138 \mathrm{~h}$, and (iii) after the sample had undergone the heat treatment. An interesting characteristic of this figure is that the total capacitance of the sample, within the low frequency range, reduces dramatically after annealing. Similar behavior has also observed by other workers for $\mathrm{CuPc}^{19}$ and $\mathrm{CoPc}^{20}$ films, and has been attributed to release of absorbed oxygen molecules from the organic layer. Furthermore, desorption of water molecules, that are known to act as molecular dipoles, may also contribute to the current observation. Capacitance in the frequency range $2 \mathrm{kHz}-1$ $\mathrm{MHz}$ seems to become nearly frequency independent reducing to a minimum value of $600 \mathrm{pF}$. From Fig. 14 it is evident that the effects of $\mathrm{O}_{2}$ doping are not completely reversed upon annealing. This is probably due to more difficult process of desorption of oxygen molecules from the bulk of the NiPc film. Nevertheless, full reversibility should be expected for a longer annealing process.

\section{SUMMARY AND CONCLUSIONS}

The dark ac conductivity of $\alpha$-NiPc films was measured as a function of frequency and temperature in sandwich structure devices employing symmetric gold ohmic contacts. It was concluded that at low temperatures a hopping-type process is the dominant conduction mechanism, whereas at higher temperatures conduction is more likely to be via a band-type transport process. Doping of NiPc films with oxygen was found to have a profound effect on its ac conductivity. In particular, at low frequency levels and over an exposure period of 12 days, ac conductivity was measured to increase by 2 orders of magnitude. Upon annealing of the oxygen doped film at $395 \mathrm{~K}$, under vacuum, conductivity was found to decrease by a factor of 27 . This reduction was ascribed to desorption of oxygen molecules from the $\alpha$-NiPc layer. Measurements of capacitance and loss tangent as a function of frequency and temperature were also performed. Obtained results were quantitatively explained by invoking the equivalent circuit model of Goswami and Goswami. ${ }^{27}$ Exposure of the sample to dry air has been found to increase the low frequency measured capacitance. The phenomenon was understood in terms of reduction in the value of internal resistance $R$ due to oxygen absorption. In addition, the observed increase is also thought to be related to the absorption of $\mathrm{H}_{2} \mathrm{O}$, which increases total polarization of $\alpha$-NiPc. Annealing of the samples in vacuum has been found to partially reverse the doping effect. It is believed that prolonged annealing can totally reverse the effects of oxygen doping.

${ }^{1}$ Z. Bao, A. J. Lovinger, and A. Dodabalapur, Appl. Phys. Lett. 69, 3066 (1996).

${ }^{2}$ M. Ishii and Y. Taga, Appl. Phys. Lett. 80, 3430 (2002).

${ }^{3}$ Rudiono, S. Okazaki, and M. Takeuchi, Thin Solid Films 334, 187 (1998).

${ }^{4}$ R. G. Gould, Coord. Chem. Rev. 156, 237 (1996).

${ }^{5}$ T. D. Anthopoulos and T. S. Shafai, Phys. Status Solidi A 181, 569 (2000).

${ }^{6}$ M. Pfeiffer, A. Beyer, T. Fritz, and K. Leo, Appl. Phys. Lett. 73, 3202 (1998).

${ }^{7}$ T. D. Anthopoulos and T. S. Shafai, J. Vac. Sci. Technol. A 20, 1 (2002).

${ }^{8}$ T. G. Abdel-Malik, R. M. Abdel-Latif, A. E. El-Samashy, and S. M. Khalil, Thin Solid Films 256, 139 (1995).

${ }^{9}$ T. D. Anthopoulos and T. S. Shafai, Phys. Status Solidi A 186, 89 (2001). 
${ }^{10}$ A. A. Ebert and H. B. Gottlieb, J. Am. Chem. Soc. 74, 2806 (1952).

${ }^{11}$ A. K. Hassan, Ph.D thesis. Keele University, U.K., 1991.

${ }^{12}$ A. Kelly and G. W. Groves, Crystallography and Crystal Defects (Longman, London, 1970).

${ }^{13}$ K. F. Schoch, J. Greggi, and T. A. Temofonte, J. Vac. Sci. Technol. A 6, 155 (1988).

${ }^{14}$ A. K. Ray, S. M. Tracey, and A. K. Hassan, IEE Proc.: Sci., Meas. Technol. 146, 205 (1999).

${ }^{15}$ B. Boudjema, G. Guillaud, M. Gamoudi, M. Maitrot, J.-J. Andre, M. Martin, and J. Simon, J. Appl. Phys. 56, 2323 (1984).

${ }^{16}$ S. R. Elliot, Adv. Phys. 36, 135 (1987).

${ }^{17}$ T. G. Abdel-Malik, R. M. Abdel-Latif, M. El-Shabasy, and M. AbdelHamid, Indian J. Phys., A 62A, 17 (1988).
${ }^{18}$ K. C. Kao, and W. Hwang, Electrical Transport in Solids (Pergamon, New York, 1981).

${ }^{19}$ R. D. Gould and A. K. Hassan, Thin Solid Films 223, 334 (1993).

${ }^{20}$ S. I. Shihub, R. D. Gould, and S. Gravano, Physica B 222, 136 (1996).

${ }^{21}$ T. S. Shafai, Ph.D. thesis, University of Keele, U.K., 1996.

${ }^{22}$ Q. Zhou and R. D. Gould, Thin Solid Films 317, 432 (1998).

${ }^{23}$ T. D. Anthopoulos and T. S. Shafai, Appl. Phys. Lett. 82, 1628 (2003).

${ }^{24}$ F. Gutmann and L. E. Lyons, Organic Semiconductors (Wiley, New York, 1967).

${ }^{25}$ A. K. Abass, A Krier, and R. A. Collins, J. Phys. D 26, 1120 (1993).

${ }^{26}$ H. S. Nalwa and P. Vasudevan, J. Mater. Sci. Lett. 2, 71 (1983).

${ }^{27}$ A. Goswami and A. P. Goswami, Thin Solid Films 16, 175 (1973). 